\title{
Antinociceptive activity of a synthetic chalcone, flavokawin B on chemical and thermal models of nociception in mice
}

\begin{abstract}
The present study examined the potential antinociceptive activity of flavokawin B (6'hydroxy-2',4'-dimethoxychalcone), a synthetic chalcone using chemical- and thermal-induced nociception models in mice. It was demonstrated that flavokawin B (FKB; 0.3, 1, 3 and 10 $\mathrm{mg} / \mathrm{kg}$ ) administered via both oral (p.o.) and intraperitoneal (i.p.) routes produced significant and dose-dependent inhibition in the abdominal constrictions induced by acetic acid, with the i.p. route producing antinociception of approximately 7-fold more potent than the p.o. route. It was also demonstrated that FKB produced significant inhibition in the two phases of the formalin-induced paw licking test. In addition, the same treatment of flavokawin B (FKB) exhibited significant inhibition of the neurogenic nociceptive induced by intraplantar injections of glutamate and capsaicin. Likewise, this compound also induced a significant increase in the response latency period to thermal stimuli in the hot plate test and its antinociceptive effect was not related to muscle relaxant or sedative action. Moreover, the antinociception effect of the FKB in the formalin-induced paw licking test and the hot plate test was not affected by pretreatment of non-selective opioid receptor antagonist, naloxone. The present results indicate that FKB produced pronounced antinociception effect against both chemical and thermal models of pain in mice that exhibited both peripheral and central analgesic activity.
\end{abstract}

Keyword: Chalcone; Flavokawin B; 6'-hydroxy-2',4'-dimethoxychalcone; Antinociceptive; Opioid system 